\title{
Genomic Dissection of Nonhost Resistance to Wheat Stem Rust in Brachypodium distachyon
}

\author{
Rafael Della Coletta, ${ }^{1,2}$ Candice N. Hirsch, ${ }^{1}$ Matthew N. Rouse, ${ }^{3,4}$ Aaron Lorenz, ${ }^{1}$ and David F. Garvin ${ }^{1,5,+}$ \\ ${ }^{1}$ Department of Agronomy and Plant Genetics, University of Minnesota, St. Paul, MN, U.S.A.; ${ }^{2}$ CAPES Foundation, Ministry of \\ Education of Brazil, Brasilia, DF, Brazil; ${ }^{3}$ USDA-ARS Cereal Disease Laboratory, St. Paul, MN, U.S.A.; ${ }^{4}$ Department of Plant \\ Pathology, University of Minnesota; and ${ }^{5}$ USDA-ARS Plant Science Research Unit, St. Paul, MN, U.S.A.
}

Accepted 24 September 2018.

\begin{abstract}
The emergence of new races of Puccinia graminis f. sp. tritici, the causal pathogen of wheat stem rust, has spurred interest in developing durable resistance to this disease in wheat. Nonhost resistance holds promise to help control this and other diseases because it is durable against nonadapted pathogens. However, the genetic and molecular basis of nonhost resistance to wheat stem rust is poorly understood. In this study, the model grass Brachypodium distachyon, a nonhost of $P$. graminis f. sp. tritici, was used to genetically dissect nonhost resistance to wheat stem rust. A recombinant inbred line (RIL) population segregating for response to wheat stem rust was evaluated for resistance. Evaluation of genome-wide cumulative single nucleotide polymorphism allele frequency differences between contrasting pools of resistant and susceptible RILs followed by molecular marker analysis identified six quantitative trait loci (QTL) that cumulatively explained $72.5 \%$ of the variation in stem rust resistance. Two of the QTLs explained $31.7 \%$ of the variation, and their interaction explained another $4.6 \%$. Thus, nonhost resistance to wheat stem rust in $B$. distachyon is genetically complex, with both major and minor QTLs acting additively and, in some cases, interacting. These findings will guide future research to identify genes essential to nonhost resistance to wheat stem rust.
\end{abstract}

Wheat stem rust, a disease caused by the fungus Puccinia graminis f. sp. tritici, has historically been one of the most devastating wheat diseases in the world (Singh et al. 2011). In the beginning of the 20th century, yield losses of approximately $10 \%$ were common in the main wheat-producing areas of the

Mention of trade names or commercial products in this publication is solely for providing specific information and does not imply recommendation or endorsement by the U.S. Department of Agriculture (USDA). USDA is an equal opportunity provider and employer. The complete nondiscrimination policy can be found on the USDA website.

${ }^{\dagger}$ Corresponding author: D. F. Garvin; E-mail: david.garvin@ars.usda.gov

Funding: R. D. Coletta was supported by a CAPES fellowship (CsF88888.076129/2013-00) from the Ministry of Education of Brazil. The research was funded by United States Department of AgricultureAgreicultural Research Service appropriated projects 5062-21000-03000D and 5062-21220-021-00D.

*The $e$-Xtra logo stands for "electronic extra" and indicates that three supplementary figures and six supplementary tables are published online.

This article is in the public domain and not copyrightable. It may be freely reprinted with customary crediting of the source. The American Phytopathological Society, 2019.
United States and reached more than $50 \%$ during epidemic years (Leonard 2001). Severe damage has also been reported in many other countries around the world (Saari and Prescott 1985). However, deploying resistant varieties and the elimination of the pathogen's alternate host barberry (Berberis spp.) led to the successful control of wheat stem rust for the last several decades (Roelfs 1985; Singh et al. 2011).

New $P$. graminis f. sp. tritici races, including the virulent Ug99 race group, have recently emerged in Africa and are overcoming the stem rust resistance in wheat varieties that are planted on more than $80 \%$ of the total wheat acreage globally (Singh et al. 2015). The first Ug99 race originated in Uganda in 1998 and, currently, 13 countries in Africa and the Middle East have confirmed the presence of at least one of the 13 known Ug99 group races (Pretorius et al. 2000; Singh et al. 2016). Further, Ug99 is spreading to major areas of wheat production, such as South Asia and Europe (Singh et al. 2015). This poses a serious economic and food security threat, given that wheat is the most widely grown cereal crop globally, has a market value of $\$ 50$ billion per year, and is both the second most important cereal in human diets and the primary source of protein in poor countries around the world (FAO 2013; WHEAT 2014). Scientists have identified at least 27 stem rust resistance genes that provide effective or partial resistance against Ug99 and its variants (Aktar-Uz-Zaman et al. 2017). However, some of the varieties resistant to $\mathrm{Ug} 99$ are susceptible to an entirely new and different $P$. graminis f. sp. tritici race (TKTTF) unrelated to Ug99. In 2013, this race caused near complete yield loss in the most widely grown variety in Ethiopia throughout the Arsi and Bale wheat-growing regions (Olivera et al. 2015). This further highlights the challenge posed by wheat stem rust as a rapidly evolving disease.

The extensive use of a single resistance gene in wheat varieties can lead to rapid evolution of new virulent $P$. graminis f. sp. tritici races (Krattinger and Keller 2016). More durable resistance can be achieved by accumulating adult plant resistance $(A P R)$ genes (Sunderwirth and Roelfs 1980). By themselves, $A P R$ genes do not confer major levels of resistance, but, when combined, they reduce disease severity and may promote more durable resistance regardless of the race of the pathogen (Knott 1988). However, few $A P R$ genes are currently known and accumulating multiple $A P R$ genes in the same wheat variety is laborious (Singh et al. 2011, 2015).

Another promising strategy to obtain durable resistance is through nonhost resistance. This type of resistance protects plants against the majority of nonadapted pathogens by employing several preformed and induced barriers (Heath 2000; Mysore and Ryu 2004). Typical nonhost resistance responses are diverse and include cell-wall thickening, cytoskeleton 
reorganization, papilla formation, production of antimicrobial compounds, and hypersensitive responses leading to cell death to impede infection by the pathogen (Mysore and Ryu 2004; Uma et al. 2011). Genes involved in these responses can be identified by various methods, such as mutant screens (Lipka et al. 2008), expression profiling (Bittel and Robatzek 2007), and molecular genetic analysis (Niks and Marcel 2009).

The model plant Brachypodium distachyon is a nonhost to wheat stem rust (Figueroa et al. 2013), although genotypic variation for nonhost resistance exists and ranges from complete immunity to pustule formation (Ayliffe et al. 2013; Bettgenhaeuser et al. 2014; Figueroa et al. 2013, 2015). Such phenotypic variation coupled with the availability of a highquality reference genome sequence make this species an ideal model for analyzing genetic components of nonhost resistance to cereal rusts (Fitzgerald et al. 2015). Previously, Ayliffe et al. (2013) and Figueroa et al. (2013) identified both pre- and postinvasion responses to cereal rusts in $B$. distachyon. The preinvasion response impedes the formation of the pathogen feeding structure (haustorium) and stops pathogen penetration without producing any visible symptoms (Holub and Cooper 2004; Mysore and Ryu 2004). The postinvasion response occurs after haustoria formation. Necrotic symptoms at the infection site may appear, because such posthaustorial responses are usually associated with programmed cell death (Mysore and Ryu 2004; Uma et al. 2011). To date, there are no studies that have documented the chromosome locations of genes responsible for nonhost rust resistance in $B$. distachyon, although genetic analyses in two different populations for wheat yellow rust resistance ( $P$. striiformis f. sp. tritici) suggested simple inheritance (Ayliffe et al. 2013).

The objective of this study was to conduct a molecular genetic analysis of nonhost resistance to wheat stem rust in $B$. distachyon, using a segregating population from a cross between two genotypes that differ in their resistance to $P$. graminis f. sp. tritici. The results of this study reveal a complex genetic architecture of nonhost resistance to wheat stem rust involving several quantitative trait loci (QTLs) that vary in their effects and act additively but can also interact with each other in some instances.

\section{RESULTS}

\section{Wheat stem rust resistance in $B$. distachyon is genetically complex.}

Two B. distachyon genotypes, $\mathrm{Bd} 21$ and $\mathrm{Bd} 2-3$, show different levels of resistance to wheat stem rust. $\mathrm{Bd} 21$ is considered resistant, based on the appearance of sparse necrotic flecking on primary leaf surfaces as the predominant disease phenotype after inoculation with $P$. graminis f. sp. tritici (Fig. 1A). In contrast, Bd2-3 is considered susceptible, with the principal disease phenotype being small to medium-sized pustules (Fig. 1B). It is noteworthy that $\mathrm{Bd} 2-3$ is one of the rare genotypes that exhibits such disease development in B. distachyon. Seedlings from $146\left(\mathrm{~F}_{6}\right.$-derived $)$ recombinant inbred lines (RILs) developed from a cross between these two genotypes were inoculated with $P$. graminis f. sp. tritici and disease development was evaluated nine days after inoculation. Disease phenotypes were recorded according to the qualitative 0 -to-4 scoring system devised for stem rust resistance in wheat (Stakman et al. 1962), in which infection types from 0 to 2 are considered resistant and 3 and 4 are considered susceptible. When two infection types were present on a leaf, the lessabundant one represented $20 \%$ or fewer of the infection sites. A total of 88 RILs were considered resistant (primary infection type 0 to 1 ), whereas 32 were considered susceptible (primary infection type 3- or 3) (Fig. 1C to G). Notably, infection types 2 and 4 were not observed. In addition, 26 RILs comprised both resistant and susceptible plants, which is indicative of residual heterozygosity for resistance genes. The ratio of resistant to susceptible RILs (88:32) did not appear to fit the 1:1 ratio expected for a single gene in a RIL population. The significant number of RILs continuing to segregate for resistance as well as the presence of a broad spectrum of intermediate infection types in the RILs (Supplementary Fig. S1) suggested that there is more than one locus for nonhost resistance to wheat stem rust segregating in B. distachyon.

\section{Multiple genomic regions are associated with wheat stem rust resistance.}

Sets of RILs that were considered the most resistant (36) or the most susceptible (20) were pooled for bulked segregant analysis (BSA) (Michelmore et al. 1991) by whole-genome resequencing. After read mapping (Supplementary Table S1), the mean genome coverage was approximately 32 and $27 \times$ for resistant and susceptible pools, respectively. The reference $B$. distachyon genome sequence is that of $\mathrm{Bd} 21$, the resistant parent of the RIL population. As such single nucleotide polymorphisms (SNPs) detected in the pools should, with rare exception, reflect the presence of susceptible parent (Bd2-3) alleles. In total, 335,946 high-quality SNPs were identified in the pools. The allele frequency of each SNP was calculated and compared between the pools, using a standard two-sided Z-test. Seven genome regions exhibited statistically significant differences in allele frequencies between the resistant and

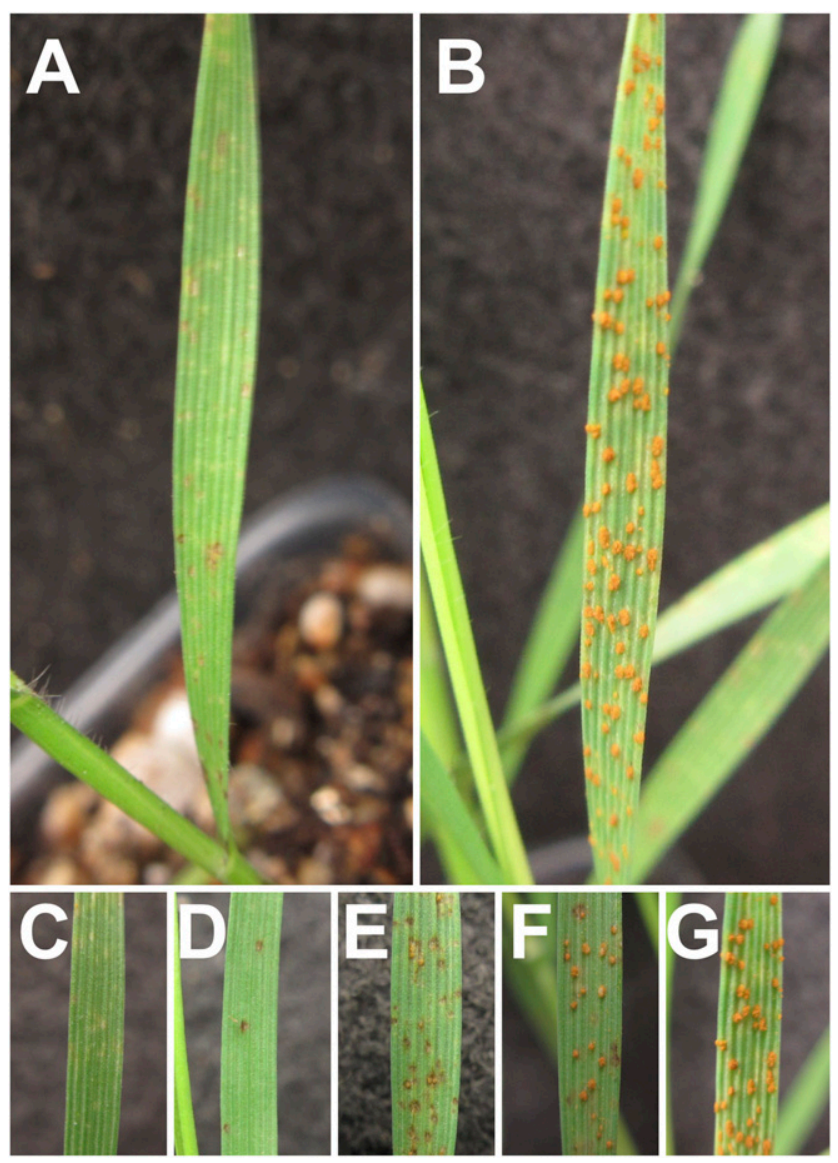

Fig. 1. Wheat stem rust disease development on inoculated primary leaves of Brachypodium distachyon inbred lines 9 days after inoculation. A, Bd21 and $\mathbf{B}, \mathrm{Bd} 2-3$. $\mathbf{C}$ to $\mathbf{E}$, Predominant infection types of resistant recombinant inbred lines (RILs): 0 (C), fleck (D), and 1 (E). F and G, Predominant infection types of susceptible RILs: $3-(\mathrm{F})$ and $3(\mathrm{G})$. 
susceptible pools (Table 1). Two regions were on chromosome 2 (BdSR1 and BdSR2), three regions were on chromosome 3 (BdSR3, BdSR4, and BdSR5), and two regions were on chromosome 4 (BdSR6 and BdSR7) (Fig. 2). BdSR4 is the largest region, at approximately $11 \mathrm{Mb}$, and spans the centromeric region of chromosome 3 . In contrast, BdSR2 represents an interval that is only $25 \mathrm{~kb}$ in length. BdSR3, BdSR5, BdSR6, and BdSR7 have sizes of $1.2 \mathrm{Mb}, 1.6 \mathrm{Mb}, 34 \mathrm{~kb}$, and $655 \mathrm{~kb}$, respectively. BdSR1 (3.4 Mb) is the only region with a high $\mathrm{Bd} 2-$
3 SNP allele frequency in the resistant but not the susceptible pool. This suggested the possibility of transgressive segregation in the population.

\section{Marker analysis validates BSA results.}

Insertion and deletion (indel) and cleaved amplified polymorphic sequence (CAPS) markers were used to obtain multilocus genotypes for the seven statistically significant regions in the entire RIL population (Table 2). Markers BdPgt-1,

Table 1. Genome coordinates of the seven genome intervals exhibiting significant allele frequency differences between resistant and susceptible RIL pools from the Bd2-3 $\times$ Bd21 RIL population

\begin{tabular}{|c|c|c|c|c|c|}
\hline Region & Chromosome & Start position & Peak allele frequency difference ${ }^{a}$ & End position & Length \\
\hline BdSR1 & $\mathrm{Bd} 2$ & 647,158 & $2,159,769$ & $4,042,643$ & $3,395,485$ \\
\hline BdSR2 & $\mathrm{Bd} 2$ & $49,044,578$ & $49,047,406$ & $49,070,196$ & 25,618 \\
\hline BdSR3 & $\mathrm{Bd} 3$ & $12,688,740$ & $13,768,730$ & $13,836,351$ & $1,147,611$ \\
\hline BdSR4 & $\mathrm{Bd} 3$ & $19,198,329$ & $20,529,541$ & $30,210,898$ & $11,012,569$ \\
\hline BdSR5 & $\mathrm{Bd} 3$ & $51,288,825$ & $52,076,013$ & $52,873,936$ & $1,585,111$ \\
\hline BdSR6 & $\mathrm{Bd} 4$ & $40,676,707$ & $40,677,265$ & $40,710,828$ & 34,121 \\
\hline BdSR7 & $\mathrm{Bd} 4$ & $46,981,209$ & $47,189,066$ & $47,637,040$ & 655,831 \\
\hline
\end{tabular}

${ }^{a}$ Middle single nucleotide polymorphism (SNP) of the most significant 15-SNP window. Numerical values are base pairs.

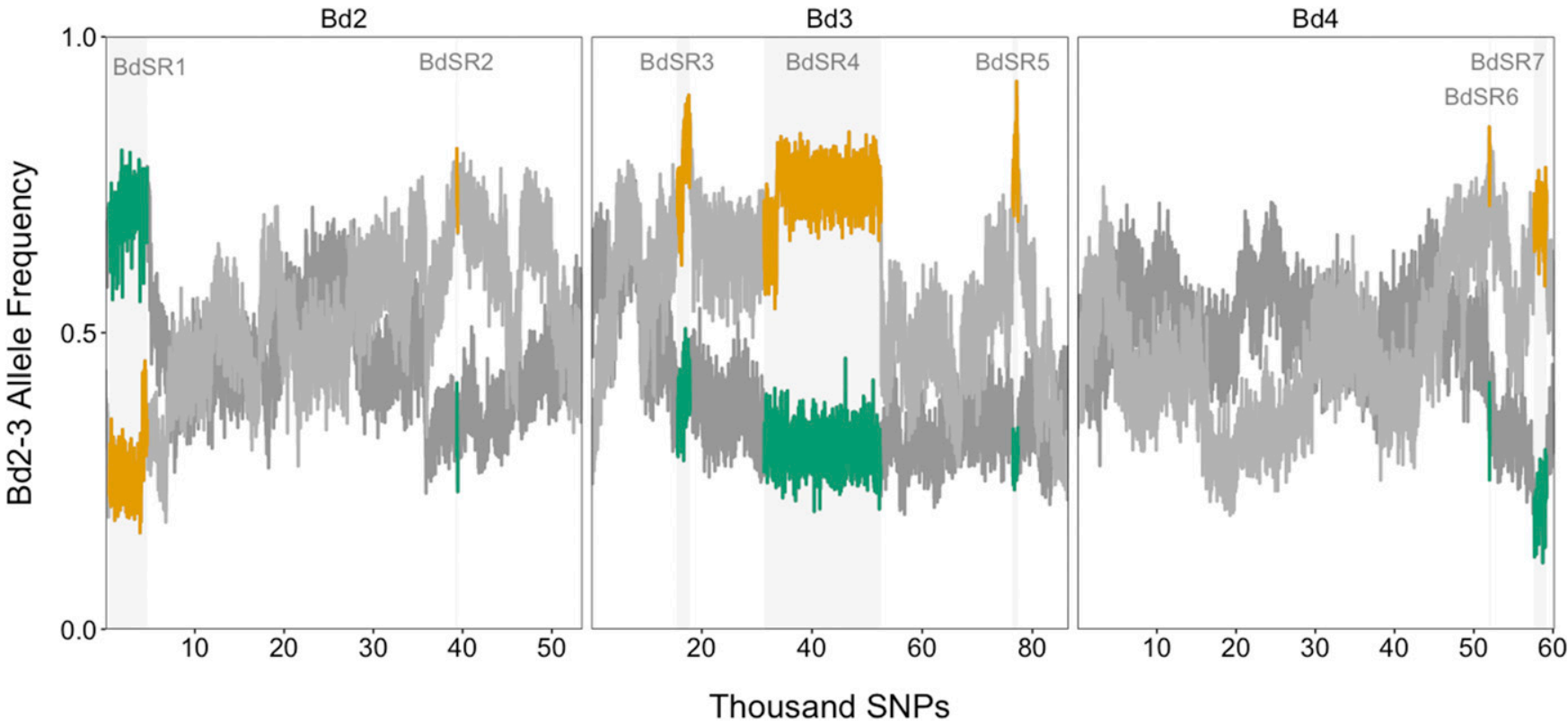

Fig. 2. Cumulative Bd2-3 single nucleotide polymorphism allele frequencies in resistant (dark gray + green lines) versus susceptible (light gray + orange lines) recombinant inbred line pools across Brachypodium distachyon chromosomes 2, 3, and 4. The orange and green highlighted regions denote the seven genome regions (BdSR1, BdSR2, BdSR3, BdSR4, BdSR5, BdSR6, and BdSR7) that exhibited statistically significant differences in allele frequencies between the two pools.

Table 2. Features of indel and CAPS markers used to obtain multilocus genotypes for stem rust resistance QTLs in the Bd2-3 $\times$ Bd 21 RIL population ${ }^{2}$

\begin{tabular}{|c|c|c|c|c|c|c|c|}
\hline \multirow{2}{*}{$\begin{array}{l}\text { Indel } \\
\text { marker }\end{array}$} & \multirow[b]{2}{*}{ Region } & \multicolumn{3}{|c|}{ Deletion } & \multirow[b]{2}{*}{ Forward primer } & \multirow[b]{2}{*}{ Reverse primer } & \multirow[b]{2}{*}{ Product size } \\
\hline & & Start & End & Size & & & \\
\hline BdPgt-1 & BdSR1 & $2,331,347$ & $2,331,416$ & 69 & GGGAGAGCAAACTGCAGAAA & CCGAGGGAGAGAGAGAGAGG & 255 \\
\hline BdPgt-2 & BdSR2 & $49,058,820$ & $49,058,835$ & 15 & CCCCATGAGTTGCATAAACA & GATTTCGTTCCTTCGCTTGA & 253 \\
\hline BdPgt-3 & BdSR3 & $13,788,486$ & $13,788,531$ & 45 & TGGCATGGAGTTTGTTGAAA & TGCCAGGATTGTAATTGTGC & 204 \\
\hline BdPgt-4 & BdSR4 & $29,440,122$ & $29,440,174$ & 52 & GCAAGCCGTTCCATTACATT & GCAGCGCAACGTGTAGTAGA & 186 \\
\hline BdPgt-6 & BdSR6 & $40,688,741$ & $40,688,778$ & 37 & CGACGATGAGTTTTCAGAGGA & CAGCTCGTCCCTCTTCTACG & 220 \\
\hline BdPgt-7 & BdSR7 & $47,060,366$ & $47,060,072$ & 6 & TAGGAGCCGATCAGACATCAC & CTCGTCCCAATTCCCAAATGG & 249 \\
\hline $\begin{array}{l}\text { CAPS } \\
\text { marker }\end{array}$ & Region & SNP & $\begin{array}{c}\text { Restriction } \\
\text { enzyme* }\end{array}$ & $\begin{array}{c}\text { Digested } \\
\text { parent }\end{array}$ & Forward primer & Reverse primer & $\begin{array}{l}\text { Undigested } \\
\text { product size }\end{array}$ \\
\hline BdPgt-5 & BdSR5 & $51,686,143$ & BsrI & $\mathrm{Bd} 21$ & CCGAGTCAAAGAAGCAAAGC & AATTTTGCTTGGCATTGGAG & 404 \\
\hline
\end{tabular}

${ }^{\text {a }}$ CAPS = cleaved amplified polymorphic sequence, $\mathrm{QTLs}=$ quantitative trait loci, $\mathrm{RIL}=$ recombinant inbred line, $\mathrm{SNP}=$ single nucleotide polymorphism

Deletion start, end, and size shown as base pair values. 
BdPgt-2, BdPgt-3, BdPgt-4, BdPgt-5, and BdPgt-6 were developed for regions BdSR1, BdSR2, BdSR3, BdSR4, BdSR5, and BdSR6, respectively. Interestingly, BdPgt-7, used for region BdSR7, was previously found to cosegregate with a major gene conferring resistance to $P$. graminis f. sp. phlei-pratense, which causes stem rust of timothy grass (Garvin 2013). To confirm that these individual markers were valid predictors for each genome region in which they reside, we first calculated the allele frequencies only for the RILs included in the BSA pools. The frequencies were comparable with those obtained from the BSA SNP allele frequency differences (Supplementary Table S2), suggesting that the markers accurately tracked their respective regions.

Subsequently, the entire RIL population was then screened with the markers, and the frequency of the resistant allele for each region was calculated (Supplementary Table S3). Resistant RILs possessed a higher frequency of resistant alleles (from 47 to 66\%), particularly for regions BdSR1, BdSR5 and BdSR7 compared with susceptible RILs (from 16 to 31\%). For the 58 most resistant RILs (those with infection types 0 or fleck), the resistant allele frequency was even higher for BdSR1 (72\%), BdSR5 (72\%) and BdSR7 (81\%). Multilocus haplotypes for each RIL are presented in Supplementary Table S4.

\section{QTLs for wheat stem rust resistance both act additively and interact.}

The seven genomic regions associated with stem rust resistance were presumed to harbor stem rust resistance QTLs. Thus, the QTLs in each region adopt the same name as the regions in which they reside, with a prefix of $\mathrm{Q}$. Given the number of QTLs, there were very few RILs with a particular multilocus haplotype. This precluded the estimation of their individual effects based on the qualitative infection type scores. Thus, infection types for the RILs were converted to numerical values using a custom perl script developed by Gao et al. (2016), with output values ranging from 0 (most resistant) to 9 (most susceptible). These values were then used for the analysis of QTL effects by analysis of variance (ANOVA) and multiple regression. After numerical conversion, the resistant RILs had values ranging between 0 and 2.67, while the susceptible RILs varied from 2.92 to 8 (Fig. 3). The 26 segregating RILs had mean values from 1.48 to 4.05 (Fig. 3). The mean numerical value of $\mathrm{Bd} 21$ was 0.02 , while $\mathrm{Bd} 2-3$ had a mean value of 5.86 (Fig. 3).

The effect of each QTL on stem rust resistance was estimated by performing a forward stepwise approach of model selection (Weisberg 2005) for ANOVA and multiple regression analysis. A scheme with the entire stepwise process is shown in Supplementary Figure S2. After four rounds of model selection, one region, BdSR2, was not confirmed to harbor a wheat stem rust resistance QTL, while the presence of QTLs in the other regions was validated. Thus, QTLs $Q B d S R 1, Q B d S R 3$, $Q B d S R 4, Q B d S R 5, Q B d S R 6$, and $Q B d S R 7$ and the QTL interactions $Q B d S R 3+Q B d S R 5$ and $Q B d S R 5+Q B d S R 7$ were selected for the final ANOVA model, because they showed statistically significant effects on resistance (Supplementary Tables S5 and S6). The total amount of variation for stem rust resistance in the RIL population that was explained by this model was $72.5 \%$. Multiple regression analysis with the significant QTL and QTL interactions was also performed to estimate their effects (Table 3). Among the QTLs, QBdSR5 has the largest effect, explaining $21.1 \%$ of variation, followed by $Q B d S R 7, Q B d S R 1, Q B d S R 3, Q B d S R 6$, and $Q B d S R 4$. For the QTL interactions, the effect of the interaction between $Q B d S R 5$ and $Q B d S R 7(4.6 \%)$ was larger than between $Q B d S R 3$ and QBdSR5 (2\%).

\section{DISCUSSION}

Nonhost resistance is proposed as a strategy to develop durable resistance to diseases in crops, but the genetic and molecular basis of this type of resistance remains poorly understood. In this study, we performed a genetic analysis of nonhost resistance to wheat stem rust using the model grass $B$. distachyon. Inoculation of the nonadapted pathogen $P$. graminis f. sp. tritici on two $B$. distachyon inbred lines resulted in differential disease symptoms, with $\mathrm{Bd} 21$ displaying only a few necrotic spots on the infected primary leaf, while large numbers of small pustules emerged from the leaf surface of Bd2-3. Variation in P. graminis $\mathrm{f}$. $\mathrm{sp}$. tritici resistance in the same $B$. distachyon lines was also reported by Figueroa et al. (2013), but Bd2-3, in their study, displayed much less pustule development than we observed. Differences in inoculum density, pathogen race, growth conditions, and the age of plants at inoculation might have contributed to milder disease development in that study. In both studies, though, the disease developed much less aggressively on $B$. distachyon than what is observed on wheat when inoculated with a compatible race.

A RIL population from a cross between $\mathrm{Bd} 21$ and $\mathrm{Bd} 2-3$ was used to investigate the genetics of nonhost resistance to $P$. graminis f. sp. tritici. After multiple replicate inoculations, each RIL was classified as homozygous resistant, homozygous susceptible, or segregating. Since the RILs were $\mathrm{F}_{6}$-derived, few remnant segregating RILs would be expected if inheritance of resistance was simple and Mendelian. Simple inheritance of nonhost resistance was postulated by Ayliffe and colleagues (2013) for wheat stripe rust (P. striiformis f. sp. tritici) in two different $B$. distachyon mapping populations in which only one or two loci appeared to be involved. However, in our study nearly $20 \%$ of the RILs were found to be segregating for resistance. This alone suggested that wheat stem rust resistance in the population was not governed by a small number of genes but, rather, was more complex.

Various permutations of BSA have been used for decades to identify loci associated with both qualitative and quantitative traits (Michelmore et al. 1991; Vikram et al. 2012). For each pool, the individuals will have a similar genetic composition for most loci except for those genetically linked with the trait of interest (Michelmore et al. 1991). Since B. distachyon has a small genome and a high-quality reference genome

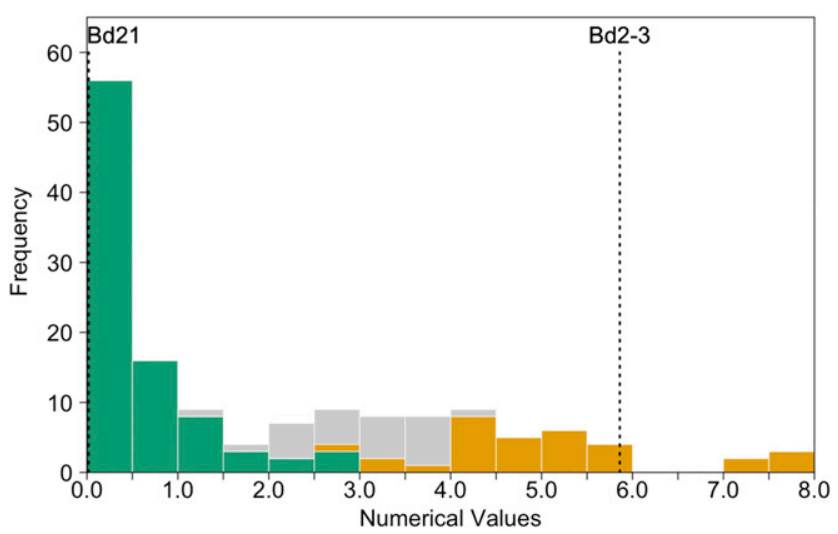

Fig. 3. Distribution of stem rust resistance of $\mathrm{Bd} 2-3 \times \mathrm{Bd} 21$ recombinant inbred lines (RILs) after conversion of qualitative 0 to 4 infection types to numerical values derived from the average of four replicates. Green bars represent homozygous resistant RILs based on initial infection-type data, while orange bars represent the contrasting homozygous susceptible RILs. Gray bars represent segregating RILs. Dashed lines indicate the mean score of the parents. The most resistant RILs displayed a converted value of 0 (corresponding to infection type 0 ), and the most susceptible RILs displayed a value of 8 (corresponding to infection type 3 ). 
assembly, whole genome sequencing-based BSA is feasible, as demonstrated by Takagi et al. (2013), and offers higher marker density than other genotyping technologies. Using this strategy in a manner similar to that employed by Haase and colleagues (2015) to map QTLs for flowering time and plant height in maize, seven genome regions associated with wheat stem rust resistance were identified in our $B$. distachyon RIL population. Subsequent QTL analysis confirmed that six of these regions possess QTLs for wheat stem rust resistance. Therefore, nonhost resistance to wheat stem rust in $B$. distachyon is complex in comparison with what has been proposed for stripe rust (Ayliffe et al. 2013).

The quantitative control of nonhost resistance to rusts has also been reported in Arabidopsis and barley. Shafiei et al. (2007) identified two QTLs related to guard-cell death and three QTLs controlling pathogen substomatal vesicle formation after challenging Arabidopsis with the wheat leaf rust pathogen (P. triticina). Thus, the six QTLs found in this study may similarly control different host defense responses that, together, provide effective nonhost resistance to wheat stem rust in B. distachyon. Jafary and colleagues (2006) identified 18 QTLs in barley involved in resistance to five nonadapted and two adapted rust species. Surprisingly, the majority of the QTLs were effective against only one rust species, which indicates pathogen specificity can occur even in nonhost resistance.

Complex genetic control of resistance was also observed in $B$. distachyon and its interaction with the adapted rust pathogen $P$. brachypodii. Barbieri et al. (2012) found that host resistance was also quantitative, and three QTLs were identified on chromosomes 2, 3, and 4. None of these QTLs appear to overlap with the QTLs found in this study, suggesting that different genes are involved in host and nonhost resistance to these different rust pathogens. In contrast, the genetically complex nonhost resistance to wheat stem rust in the RIL population employed in this study is interesting because the same RIL population appears to segregate for just a single major gene for timothy grass stem rust resistance and this gene's location is coincident with the 655-kb genome interval defined by $Q B d S R 7$, identified in this study (Garvin 2013). This suggests the possibility that this gene and $Q B d S R 7$ involve the action of the same gene. If so, it would imply that the relative magnitude of the same nonhost resistance gene can vary depending on the pathogen. Genetic analysis with more stem rust formae speciales and other rust pathogens may expand our knowledge of this issue.

The findings of this study highlight important aspects of $B$. distachyon genetics. QBdSR6 was the smallest identified QTL, defined by a genome interval just $34 \mathrm{~kb}$ in length. This may reflect the high recombination rate of $B$. distachyon (Huo et al. 2011). Previously, a gene for barley stripe mosaic virus resistance was mapped to a $23-\mathrm{kb}$ interval, using a $B$. distachyon RIL population of comparable size (Cui et al. 2012). In contrast, $Q B d S R 4$ is within an $11-\mathrm{Mb}$ interval. Since it spans the centromere of chromosome 3 , its large size may be explained by the low recombination rate in centromeric regions of the $B$. distachyon genome (International Brachypodium Initiative 2010).

An examination of gene content within the QTL intervals provides new insights into the potential mechanistic basis of nonhost resistance to stem rust in $B$. distachyon. Only four genes are present in the smallest interval, QBdSR6, and three of them have functional annotations. In this interval, the gene Bradi4g35301 is an uncharacterized conserved protein containing a GRAM domain and is homologous to the Arabidopsis $B A G P 1$ gene, which mediates resistance to fungal pathogens (Li et al. 2016). This QTL interval also contains the gene Bradi4g35317 that encodes a leucine-rich repeat-containing protein with an NB-ARC domain, which is typically related to a hypersensitive-defense response in plants (van der Biezen and Jones 1998). Further, the interval contains a gene (Bradi4g35330) encoding a Rho GTPase-binding protein (formin) that is homologous to the Arabidopsis AFH1 gene. AFH1 is involved in actin cytoskeleton organization (Michelot et al. 2006), which is considered an early plant immune response system to pathogen infection (Day et al. 2011; Takemoto et al. 2003). Thus, while it is not possible to determine which gene or genes in each QTL interval contribute to nonhost resistance, it is tempting to speculate that genes related to both pre- and postinvasion defense responses may play a role in $B$. distachyon nonhost resistance to $P$. graminis f. sp. tritici. Developing mapping populations by selfing RILs segregating for an individual QTL would be an approach to fine-map these genes in larger QTL intervals (Tuinstra et al. 1997). For the smaller QTLs such as QBdSR6, QTL identification may be achieved by genome editing (Luo et al. 2016), by knocking out candidate genes in $\mathrm{Bd} 21$ and assessing the effect on resistance.

The results of this study indicate that nonhost resistance to wheat stem rust in $B$. distachyon is a quantitative trait in which each gene contributes small effects to achieve a high level of resistance. The resistant allele for one of the six QTLs, $Q B d S R 1$, is derived from the susceptible parent $\mathrm{Bd} 2-3$. Its effect on resistance is minor, given both that $\mathrm{Bd} 2-3$ is quite susceptible to $P$. graminis $\mathrm{f}$. sp. tritici and that the amount of variation for stem rust resistance that it contributes is only $4 \%$. However, it is interesting that the frequency of $Q B d S R l$ in the pool of resistant RILs used for BSA is four times greater than in the susceptible pool of RILs. This indicates that it is exerting a large enough effect to permit visual discrimination of its effect on enhancing resistance. Further, the observed transgressive segregation toward greater susceptibility than $\mathrm{Bd} 2-3$ in some of the RILs suggests that its absence may explain this finding.

To better understand the effects of each QTL on nonhost resistance, markers were developed for each QTL and were employed to obtain multilocus QTL genotypes for the entire RIL population. This permitted ANOVA and multiple regression

Table 3. Multiple regression analysis with significant individual quantitative trait loci (QTLs) and QTL interactions to obtain the contribution of each QTL to stem rust resistance in the $\mathrm{Bd} 2-3 \times \mathrm{Bd} 21 \mathrm{RIL}$ population

\begin{tabular}{|c|c|c|c|c|c|}
\hline Variable $^{\mathbf{a}}$ & Estimate & Standard error & $t$ value & $\operatorname{Pr}(>|t|)$ & $\%$ variation explained \\
\hline (Intercept) & 6.0437 & 0.3018 & 20.027 & $<2 \mathrm{e}-16$ & - \\
\hline$Q B d S R 1 * * *$ & -1.0371 & 0.2694 & -3.849 & 0.000221 & 4.2 \\
\hline$Q B d S R 3 *$ & -0.884 & 0.3943 & -2.242 & 0.027408 & - \\
\hline$Q B d S R 4 *$ & -0.6706 & 0.2665 & -2.516 & 0.013642 & 1.8 \\
\hline$Q B d S R 5 * * *$ & -3.7056 & 0.4072 & -9.1 & $2.13 \mathrm{E}-14$ & 21.1 \\
\hline QBdSR6 *** & -0.8756 & 0.2506 & -3.494 & 0.000741 & 3.4 \\
\hline$Q B d S R 7 * * *$ & -2.7742 & 0.3947 & -7.028 & $3.90 \mathrm{E}-10$ & 10.6 \\
\hline$Q B d S R 3: Q B d S R 5 *$ & 1.3264 & 0.5053 & 2.625 & 0.010175 & 2 \\
\hline$Q B d S R 5: Q B d S R 7 * * *$ & 2.0238 & 0.5027 & 4.026 & 0.000118 & 4.6 \\
\hline
\end{tabular}

a One asterisk (*) indicates $P<0.05$, two (**) $P<0.01$, and three (***) $P<0.001$. 
analysis to be performed using qualitative phenotypic scores that had been converted to a continuous numerical scale, using the same software developed to identify QTLs conferring resistance to leaf rust in wheat (Gao et al. 2016). This analysis revealed that $72.5 \%$ of the total variation in stem rust resistance was explained by six QTLs and by the QTL interactions $Q B d S R 3+Q B d S R 5$ and $Q B d S R 5+Q B d S R 7$. Interestingly, $Q B d S R 3$ was only considered significant in ANOVA because of its interaction with $Q B d S R 5$, which suggests that epistasis also plays a role in nonhost resistance. Epistatic interactions were also reported in other nonhost interactions. For instance, Zhang et al. (2009) detected epistasis among three QTLs that confer nonhost resistance of wild lettuce (Lactuca saligna) to the nonadapted fungal pathogen Bremia lactucae and Lee et al. (2014) demonstrated that complimentary dominant epistasis may play a role in the cell-death response of the nonhost pepper against the potato late blight pathogen (Phytophtora infestans). Interestingly, epistasis has also been reported among wheat QTLs conferring adult plant resistance to Ug99 (Rouse et al. 2014).

Although it was not possible to look at all possible QTL interactions due to the modest population size, two QTLs deserve particular attention. These QTLs, QBdSR5 and $Q B d S R 7$, explain $31.7 \%$ of the variation in stem rust resistance and also have the largest effect for QTL interactions. If these QTLs can be isolated, future experiments to transfer them into wheat would permit an evaluation of the effectiveness of foreign nonhost resistance gene introduction as a strategy for enhancing wheat stem rust resistance.

Nonhost resistance has great potential to provide crops with durable and broad-spectrum resistance against important diseases, such as wheat stem rust. To our knowledge, this is the first study in B. distachyon that has mapped the locations of loci related to nonhost resistance to wheat stem rust. Resistance to wheat stem rust appears to be complex and involves additive and epistatic effects in the population evaluated in this study. Further studies to identify genes that underlie these QTLs will provide a better understanding of the molecular mechanisms that control nonhost resistance to stem rust in B. distachyon. Additionally, they ultimately will provide opportunities introduce the genes into wheat as a means of adding a new and durable layer of resistance to this serious disease.

\section{MATERIALS AND METHODS}

\section{Plant materials.}

Two B. distachyon inbred lines, Bd21 (United States Department of Agriculture (USDA) National Plant Germplasm System (NPGS) accession number W6 36678) and Bd2-3 (USDA NPGS accession number W6 46202) (Garvin 2016), and a $F_{6: 8}$ RIL population consisting of 146 RILs from a cross between them were used for this study.

\section{Pathogen methods.}

The $P$. graminis f. sp. tritici isolate 78-21-BB463 (P. graminis f. sp. tritici 4a) (Zambino et al. 2000) was used for disease evaluations. To obtain spores for inoculations, seeds of wheat cultivar McNair were planted, were placed in a glass deli cooler $\left(5 \pm 3^{\circ} \mathrm{C}\right)$ for 4 days, and were then transferred to a growth chamber (Conviron, model E8VH) with the following settings: $21^{\circ} \mathrm{C}, 14-\mathrm{h}$ day length, and a light intensity of $300 \mu \mathrm{mol} \mathrm{m} \mathrm{m}^{-2} \mathrm{~s}^{-1}$. After 8 days, developing seedlings were fertilized and were treated with a solution of maleic hydrazide at a final concentration of $0.003 \%$. Five days later, wheat seedlings were inoculated, with $2 \mathrm{mg}$ of $P$. graminis f. sp. tritici 4a spores suspended in $600 \mu \mathrm{l}$ of Soltrol 170 oil, by spraying the solution onto seedlings, using a fine pneumatic mister. One hour after inoculation, plants were transferred to a plexiglass chamber with an attached humidifier inside a growth chamber. Plants were misted for 2 -min periods every $12 \mathrm{~min}$ at $20^{\circ} \mathrm{C}$ for $17.5 \mathrm{~h}$ in the dark. Lights were then turned on and the temperature was raised to $22^{\circ} \mathrm{C}$ for $2 \mathrm{~h}$. Misting was then stopped, and plants were transferred back to the original growth chamber $3 \mathrm{~h}$ later. Twelve days after inoculation, McNair leaves were covered with uredinia producing urediniospores, which were harvested, were transferred to gelatin capsules, and were stored inside cryovials in a deli cooler $\left(5 \pm 3^{\circ} \mathrm{C}\right)$ until use for inoculations.

\section{Inoculation of $B$. distachyon RILs.}

Five seeds per RIL were planted in separate quadrants of $2.25 \times 2.5 \times 2.5$-inch pots, so each pot contained four RILs. Parents were planted to serve as controls (one quadrant of each parent per six pots). The pots were placed in trays (six to a tray), were placed in a deli cooler $\left(5 \pm 3^{\circ} \mathrm{C}\right)$ for 5 days, and were then transferred to a growth chamber with the following conditions: $24^{\circ} \mathrm{C}, 14$-h day length, and a light intensity of $150 \mu \mathrm{mol} \mathrm{m} \mathrm{m}^{-2} \mathrm{~s}^{-1}$. Seven days later, seedlings were at the one- to two-leaf stage and were ready for inoculation. Seedlings that showed a lag in development were removed to ensure all plants were at the same developmental stage. The inoculation process was as described previously for the spore increases, except for the number of spores. Optimal inoculation conditions were established and involved suspending approximately $5 \mathrm{mg}$ of freshly harvested spores (between 1 and 6 days old) in $500 \mu \mathrm{l}$ of Soltrol 170 oil in a gelatin capsule and spraying one such capsule onto each tray of $B$. distachyon seedlings. After overnight misting, plants were transferred back to a growth chamber and were maintained until they were evaluated for disease symptoms. The RILs were inoculated and evaluated in four separate replicates planted over time.

\section{Disease evaluation.}

Nine days after inoculation, plants were evaluated for stem rust symptoms by recording infection types on the inoculated primary leaves. The scoring system used to determine infection type was based on the standard wheat stem rust seedling scoring method (Stakman et al. 1962). If more than one infection type was present, the predominant one was recorded first, followed by the second one. For example, a ';3-' score indicates that the prevalent infection type is (fleck) but a few small type 3 pustules are present. The infection type was recorded for each individual plant for each RIL, in order to obtain the consensus infection type for each RIL in each inoculation. Moreover, infection types from each inoculation were converted to numerical values using a custom perl script "convert_rust_reading.seedling.pl" developed by Gao et al. (2016) and freely available on GitHub. The numerical scale ranges from 0 (most resistant) to 9 (most susceptible). Thus, mean resistance scores for each of these qualitative and numerical disease rating systems across the four inoculations were obtained for each RIL for subsequent genetic analysis.

\section{BSA.}

Plants were cut back, following the last inoculation replicate, to allow growth of pathogen-free leaves. Ten days later, when emerging leaves were between 2 and 3 inches long, leaf tissue for BSA was harvested. Thirty-six RILs were selected for the resistant pool and 20 RILs were selected for the susceptible pool, using resistance data from the multiple replicate inoculations. Three leaves per RIL were harvested for the resistant group, while five leaves per RIL were harvested for the susceptible group. This was done to have two tissue pools with a similar mass for grinding. The leaves for each RIL chosen were of equal size and were then combined. The leaves for each pool 
were combined and ground in liquid nitrogen, and DNA was extracted using the Qiagen DNeasy plant mini kit, according to the manufacturer instructions. Next generation sequencing library preparation and sequencing were completed by the University of Minnesota Genomics Center with DNA input of 400 and $350 \mathrm{ng}$ for resistant and susceptible pools, respectively. Library preparation was completed with the TruSeq nano kit, and sequencing was conducted on an Illumina HiSeq 2500 (rapid mode) to obtain 100-bp paired-end reads.

\section{Bioinformatics analysis of BSA data.}

After sequencing, the quality of raw reads was initially evaluated with FastQC v0.11.2, and those containing universal and Illumina-specific sequencing adapters were removed, using CutAdapt v.1.8.1 (Martin 2011), from both ends, keeping reads with a minimum length of $50 \mathrm{bp}$. FASTX toolkit v.0.0.14 was used for further quality control, which involved discarding low complexity sequences using the fastx_artifacts_filter, trimming bases with low quality from the end of the sequences, and discarding reads shorter than $50 \mathrm{bp}$ with fastq_quality_trimmer. A custom python script, developed by $\mathrm{M}$. Vermaat and freely available at GitHub, was used to sync paired-end reads. These reads were then mapped to the reference $\mathrm{Bd} 21 \mathrm{~B}$. distachyon genome sequence (International Brachypodium Initiative 2010) (B. distachyon v3.1, available online at Phytozome 12) with Bowtie2 v2.2.4 (Langmead and Salzberg 2012) in the end-toend mode with the very-sensitive option set.

SNPs were called, with SAMtools v1.2 mpileup and BCFtools v1.2 (Li 2011) "bcftools call" with the consensuscaller method and were then filtered using the "vcfutils.pl varFilter" program from SAMTools v1.2, keeping only SNPs with high mapping quality (option '-Q 30') and with read depth between 10 and 60 reads. The filter program of BCFTools v1.2 was also used to remove SNPs within 5 bp of an indel (option '-g $5^{\prime}$ ), to exclude sites that have less than 10 reads supporting the reference and the alternate alleles and, also, to remove SNPs with low genotype quality. Then, $\mathrm{Bd} 2-3$ allele frequencies were calculated, in each pool, based on read depth. To identify regions with statistically significant differences between allele frequencies of the two pools, a custom $R$ script developed and described by Haase et al. (2015) was used to calculate standard two-sided Z-tests averaged in windows of 15 SNPs. After conversion of the $P$ values to $-\log _{10}$, regions above the threshold of 3.42, which represents the $1 \%$ outlier threshold, were called significantly different. Graphs of cumulative SNP frequencies in each chromosome were made with 20 SNP windows (rolling every five SNPs) in $R$ v3.1.2, using a modified script available on GitHub of the Genomics, Evolution and Development Lab at Michigan State University.

\section{Marker analysis.}

Resequencing data of the susceptible parent $\mathrm{Bd} 2-3$, produced by the United States Department of Energy Joint Genome Institute website, was used to identify sequence differences between the parents, and subsequently, to generate molecular markers to represent the regions identified as statistically different by the BSA bioinformatics analysis. After quality control, $\mathrm{Bd} 2-3$ reads were mapped to the reference genome using Bowtie2 v2.2.4. Pindel v0.2.5b6 (Ye et al. 2009) was used to identify deletions for development of indel markers, and SAMtools v1.2 and BCFtools v1.2 were used to identify homozygous SNPs in the $\mathrm{Bd} 2-3$ genome relative to $\mathrm{Bd} 21$ for CAPS marker development. Once sequence variants were identified, $250 \mathrm{bp}$ of Bd21 DNA sequence was extracted from each side of a deletion or a SNP in Bd2-3. To identify restriction site differences between the parents for CAPS markers, the online software NEBcutter v2.0 (Vincze et al. 2003) from New
England Laboratories was used. Polymerase chain reaction (PCR) primers flanking the mutations were designed with Primer3 and were validated using the local version of reverse electronic PCR (re-PCR) v.2.3.12 (Rotmistrovsky et al. 2004; Schuler 1997).

DNA from individual RILs was used to score the markers in the RIL population representing each statistically different region identified by BSA analysis. Indel markers were amplified using the SYBR green Kit (Applied Biosystems) and were subjected to the following melt curve analysis, using the StepOnePlus real-time PCR system (Applied Biosystems): initial denaturation at $95^{\circ} \mathrm{C}$ for $15 \mathrm{~s}$, annealing at $52^{\circ} \mathrm{C}$ for $1 \mathrm{~min}$ (recording DNA quantification every $0.3^{\circ} \mathrm{C}$ ), and denaturation again at $95^{\circ} \mathrm{C}$. The melt curves of each RIL were compared with the melt curves of the parents to determine its genotype, based on differences in melting temperatures of the amplicons from the parents (Supplementary Fig. S3). Vertical acrylamide gel electrophoresis was used to score markers when melting curve analysis did not differentiate the alleles. Acrylamide gel electrophoresis and silver staining of amplicons were conducted as described in Liu and Anderson (2003), and markers were scored based on size differences of PCR products. RIL population screening with CAPS markers was done after PCR amplification, enzymatic product digestion, and $2 \%$ agarose gel electrophoresis.

\section{QTL effects.}

Mean numerical conversion values for resistance levels were used to estimate the effect of each genomic region of interest on stem rust resistance by performing ANOVA and multiple regression analysis. A forward stepwise approach of model selection (Weisberg 2005) for ANOVA was done with the 'Anova' function of the $R$ package " $c a r$ " with the type III sum of squares option selected to account for QTL interactions. RILs that were heterozygous at a SNP or with segregating phenotypes were removed from analysis. First, seven ANOVA models containing one of the seven putative QTLs were analyzed and only those that were statistically significant $(P<0.05)$ were selected. Second, 21 ANOVA models representing all possible pairwise interactions among the seven putative QTLs were fit. Only marker interactions with significant effects were retained for further analysis. Next, the significant individual putative QTLs from the first step and the significant QTL interactions from the second step were added in the same model and another ANOVA was performed. Only the significant terms in the model were retained, with the variation attributed to nonsignificant terms being pooled with the error variance. Finally, a new ANOVA model was developed in which all terms (both main effects and interactions) were significant. Multiple regression analysis of this final model was also carried out to obtain the contribution of each significant QTL and QTL interaction to stem rust resistance.

\section{ACKNOWLEDGMENTS}

The authors thank Z. Blankenheim for excellent technical assistance. The authors acknowledge the Minnesota Supercomputing Institute (MSI) at the University of Minnesota for providing resources that contributed to the research results reported within this paper.

\section{LITERATURE CITED}

Aktar-Uz-Zaman, M., Tuhina-Khatun, M., Hanafi, M. M., and Sahebi, M. 2017. Genetic analysis of rust resistance genes in global wheat cultivars: An overview. Biotechnol. Biotechnol. Equip. 31:431-445.

Ayliffe, M., Singh, D., Park, R., Moscou, M., and Pryor, T. 2013. Infection of Brachypodium distachyon with selected grass rust pathogens. Mol. Plant-Microbe Interact 26:946-957. 
Barbieri, M., Marcel, T. C., Niks, R. E., Francia, E., Pasquariello, M., Mazzamurro, V., Garvin, D. F., and Pecchioni, N. 2012. QTLs for resistance to the false brome rust Puccinia brachypodii in the model grass Brachypodium distachyon L. Genome 55:152-163.

Bettgenhaeuser, J., Gilbert, B., Ayliffe, M., and Moscou, M. J. 2014 Nonhost resistance to rust pathogens-A continuation of continua. Front. Plant Sci. 5:664.

Bittel, P., and Robatzek, S. 2007. Microbe-associated molecular patterns (MAMPs) probe plant immunity. Curr. Opin. Plant Biol. 10:335-341.

Cui, Y., Lee, M. Y., Huo, N., Bragg, J., Yan, L., Yuan, C., Li, C., Holditch, S. J., Xie, J., Luo, M. C., Li, D., Yu, J., Martin, J., Schackwitz, W., Gu, Y. Q., Vogel, J. P., Jackson, A. O., Liu, Z., and Garvin, D. F. 2012. Fine mapping of the Bsrl barley stripe mosaic virus resistance gene in the model grass Brachypodium distachyon. PLoS One 7:e38333.

Day, B., Henty, J. L., Porter, K. J., and Staiger, C. J. 2011. The pathogenactin connection: A platform for defense signaling in plants. Annu. Rev. Phytopathol. 49:483-506.

FAO. 2013. World Food and Agriculture. In: FAO Statistical Yearbook Food and Agriculture Organization of the United Nations, Rome. Published online. http://www.fao.org/docrep/018/i3107e/i3107e00.htm

Figueroa, M., Alderman, S., Garvin, D. F., and Pfender, W. F. 2013. Infection of Brachypodium distachyon by formae speciales of Puccinia graminis: Early infection events and host-pathogen incompatibility. PLoS One 8:e56857.

Figueroa, M., Castell-Miller, C. V., Li, F., Hulbert, S. H., and Bradeen, J. M. 2015. Pushing the boundaries of resistance: Insights from Brachypodium-rust interactions. Front. Plant Sci. 6:558.

Fitzgerald, T. L., Powell, J. J., Schneebeli, K., Hsia, M. M., Gardiner, D. M., Bragg, J. N., McIntyre, C. L., Manners, J. M., Ayliffe, M., Watt, M., Vogel, J. P., Henry, R. J., and Kazan, K. 2015. Brachypodium as an emerging model for cereal-pathogen interactions. Ann. Bot. 115: 717-731.

Gao, L., Turner, M. K., Chao, S., Kolmer, J., and Anderson, J. A. 2016 Genome wide association study of seedling and adult plant leaf rust resistance in elite spring wheat breeding lines. PLoS One 11: e0148671.

Garvin, D. F. 2013. Genome analysis of nonhost resistance to stem rust in Brachypodium. Page 59 in: Proceedings of the $12^{\text {th }}$ International Wheat Genetics Symposium. Yokohama, Japan.

Garvin, D. F. 2016. Brachypodium distachyon genetic resources. Pages 183-195 in: Genetics and Genomics of Brachypodium. J. P. Vogel, ed. Springer, Cham.

Haase, N. J., Beissinger, T., Hirsch, C. N., Vaillancourt, B., Deshpande, S. Barry, K., Buell, C. R., Kaeppler, S. M., and de Leon, N. 2015. Shared genomic regions between derivatives of a large segregating population of maize identified using bulked segregant analysis sequencing and traditional linkage analysis. G3 (Bethesda) 5:1593-1602

Heath, M. C. 2000. Nonhost resistance and nonspecific plant defenses Curr. Opin. Plant Biol. 3:315-319.

Holub, E. B., and Cooper, A. 2004. Matrix, reinvention in plants: How genetics is unveiling secrets of non-host disease resistance. Trends Plant Sci. 9:211-214.

Huo, N., Garvin, D. F., You, F. M., McMahon, S., Luo, M. C., Gu, Y. Q. Lazo, G. R., and Vogel, J. P. 2011. Comparison of a high-density genetic linkage map to genome features in the model grass Brachypodium distachyon. Theor. Appl. Genet. 123:455-464.

International Brachypodium Initiative. 2010. Genome sequencing and analysis of the model grass Brachypodium distachyon. Nature 463 : 763-768.

Jafary, H., Szabo, L. J., and Niks, R. E. 2006. Innate nonhost immunity in barley to different heterologous rust fungi is controlled by sets of resistance genes with different and overlapping specificities. Mol. PlantMicrobe Interact 19:1270-1279.

Knott, D. R. 1988. Using polygenic resistance to breed for stem rust resistance in wheat. Pages 39-47 in: Breeding Strategies for Resistance to the Rusts of Wheat. Simmonds, N. W., and Rajaram, S., eds. CIMMYT, D.F., Mexico.

Krattinger, S. G., and Keller, B. 2016. Molecular genetics and evolution of disease resistance in cereals. New Phytol. 212:320-332.

Langmead, B., and Salzberg, S. L. 2012. Fast gapped-read alignment with Bowtie 2. Nat. Methods 9:357-359.

Lee, H. A., Kim, S. Y., Oh, S. K., Yeom, S. I., Kim, S. B., Kim, M. S., Kamoun, S., and Choi, D. 2014. Multiple recognition of RXLR effectors is associated with nonhost resistance of pepper against Phytophthora infestans. New Phytol. 203:926-938.

Leonard, K. J. 2001. Stem rust-Future enemy? Pages 119-146 in: Stem Rust of Wheat: from Ancient Enemy to Modern Foe. P. D. Peterson, ed. APS Press, St. Paul, MN, U.S.A.
Li, H. 2011. A statistical framework for SNP calling, mutation discovery, association mapping and population genetical parameter estimation from sequencing data. Bioinformatics 27:2987-2993.

Li, Y., Kabbage, M., Liu, W., and Dickman, M. B. 2016. Aspartyl proteasemediated cleavage of BAG6 is necessary for autophagy and fungal resistance in plants. Plant Cell 28:233-247.

Lipka, U., Fuchs, R., and Lipka, V. 2008. Arabidopsis non-host resistance to powdery mildews. Curr. Opin. Plant Biol. 11:404-411.

Liu, S., and Anderson, J. A. 2003. Marker assisted evaluation of fusarium head blight resistant wheat germplasm. Crop Sci. 43:760-766.

Luo, M., Gilbert, B., and Ayliffe, M. 2016. Applications of CRISPR/Cas9 technology for targeted mutagenesis, gene replacement and stacking of genes in higher plants. Plant Cell Rep. 35:1439-1450.

Martin, M. 2011. Cutadapt removes adapter sequences from highthroughput sequencing reads. EMBnet J. 17:10-12.

Michelmore, R. W., Paran, I., and Kesseli, R. V. 1991. Identification of markers linked to disease-resistance genes by bulked segregant analysis: A rapid method to detect markers in specific genomic regions by using segregating populations. Proc. Natl. Acad. Sci. U.S.A. 88:9828-9832.

Michelot, A., Derivery, E., Paterski-Boujemaa, R., Guérin, C., Huang, S., Parcy, F., Staiger, C. J., and Blanchoin, L. 2006. A novel mechanism for the formation of actin-filament bundles by a nonprocessive formin. Curr. Biol. 16:1924-1930.

Mysore, K. S., and Ryu, C. M. 2004. Nonhost resistance: How much do we know? Trends Plant Sci. 9:97-104

Niks, R. E., and Marcel, T. C. 2009. Nonhost and basal resistance: How to explain specificity? New Phytol. 182:817-828

Olivera, P., Newcomb, M., Szabo, L. J., Rouse, M., Johnson, J., Gale, S., Luster, D. G., Hodson, D., Cox, J. A., Burgin, L., Hort, M., Gilligan, C. A., Patpour, M., Justesen, A. F., Hovmøller, M. S., Woldeab, G., Hailu, E., Hundie, B., Tadesse, K., Pumphrey, M., Singh, R. P., and Jin, Y. 2015. Phenotypic and genotypic characterization of race TKTTF of Puccinia graminis f. sp. tritici that caused a wheat stem rust epidemic in southern Ethiopia in 2013-14. Phytopathology 105:917-928.

Pretorius, Z. A., Singh, R. P., Wagoire, W. W., and Payne, T. S. 2000 Detection of virulence to wheat stem rust resistance gene $\mathrm{Sr} 31$ in Puccinia graminis f. sp. tritici in Uganda. Plant Dis. 84:203.

Roelfs, A. P. 1985. Wheat and rye stem rust. Pages 22-57 in: The Cereal Rusts, Vol I: Diseases, Distribution, Epidemiology, and Control. W. R. Bushnell and A. P. Roelfs, eds., Academic Press, Orlando, FL, U.S.A.

Rotmistrovsky, K., Jang, W., and Schuler, G. D. 2004. A web server for performing electronic PCR. Nucleic Acids Res. 32:W108-W112.

Rouse, M. N., Talbert, L. E., Singh, D., and Sherman, J. D. 2014 Complementary epistasis involving Sr12 explains adult plant resistance to stem rust in Thatcher wheat (Triticum aestivum L.). Theor. Appl. Genet. 127:1549-1559.

Saari, E. E., and Prescott, J. M. 1985. World distribution in relation to economic losses. Pages 257-293 in: The Cereal Rusts, Vol I: Diseases, Distribution, Epidemiology, and Control. W. R. Bushnell and A. P. Roelfs, eds., Academic Press, Orlando, FL, U.S.A.

Schuler, G. D. 1997. Sequence mapping by electronic PCR. Genome Res. 7: $541-550$

Shafiei, R., Hang, C., Kang, J. G., and Loake, G. J. 2007. Identification of loci controlling non-host disease resistance in Arabidopsis against the leaf rust pathogen Puccinia triticina. Mol. Plant Pathol. 8:773-784.

Singh, R. P., Hodson, D. P., Huerta-Espino, J., Jin, Y., Bhavani, S., Njau, P., Herrera-Foessel, S., Singh, P. K., Singh, S., and Govindan, V. 2011. The emergence of $\mathrm{Ug} 99$ races of the stem rust fungus is a threat to world wheat production. Annu. Rev. Phytopathol. 49:465-481.

Singh, R. P., Hodson, D. P., Jin, Y., Lagudah, E. S., Ayliffe, M. A., Bhavani, S., Rouse, M. N., Pretorius, Z. A., Szabo, L. J., Huerta-Espino, J., Basnet, B. R., Lan, C., and Hovmøller, M. S. 2015. Emergence and spread of new races of wheat stem rust fungus: Continued threat to food security and prospects of genetic control. Phytopathology 105:872-884.

Singh, R. P., Singh, P. K., Rutkoski, J., Hodson, D. P., He, X., Jørgensen, L. N., Hovmøller, M. S., and Huerta-Espino, J. 2016. Disease impact on wheat yield potential and prospects of genetic control. Annu. Rev. Phytopathol. 54:303-322.

Stakman, E. C., Stewart, D. M., and Loegering, W. Q. 1962. Identification of physiologic races of Puccinia graminis var. tritici. United States Department of Agriculture, Agricultural Research Service E-617, Washington, DC.

Sunderwirth, S. D., and Roelfs, A. P. 1980. Greenhouse evaluation of the adult plant resistance of $S r 2$ to wheat stem rust. Phytopathology 70 634-637.

Takagi, H., Abe, A., Yoshida, K., Kosugi, S., Natsume, S., Mitsuoka, C., Uemura, A., Utsushi, H., Tamiru, M., Takuno, S., Innan, H., Cano, L. M., Kamoun, S., and Terauchi, R. 2013. QTL-seq: Rapid mapping of 
quantitative trait loci in rice by whole genome resequencing of DNA from two bulked populations. Plant J. 74:174-183.

Takemoto, D., Jones, D. A., and Hardham, A. R. 2003. GFP-tagging of cell components reveals the dynamics of subcellular re-organization in response to infection of Arabidopsis by oomycete pathogens. Plant J. 33: 775-792.

Tuinstra, E. G., Ejeta, G., and Goldsbrough, P. B. 1997. Heterogeneous inbred family (HIF) analysis: A method for developing near-isogenic lines that differ at a quantitative trait loci. Theor. Appl. Genet. 95: 1005-1011.

Uma, B., Rani, T. S., and Podile, A. R. 2011. Warriors at the gate that never sleep: Non-host resistance in plants. J. Plant Physiol. 168:2141-2152.

van der Biezen, E. A., and Jones, J. D. 1998. The NB-ARC domain: A novel signalling motif shared by plant resistance gene products and regulators of cell death in animals. Curr. Biol. 8:R226-R228.

Vikram, P., Swamy, B. P. M., Dixit, S., Ahmed, H., Sta Cruz, M. T., Singh, A. K., Ye, G., and Kumar, A. 2012. Bulk segregant analysis: An effective approach for mapping consistent-effect drought grain yield QTLs in rice. Field Crops Res. 134:185-192.

Vincze, T., Posfai, J., and Roberts, R. J. 2003. NEBcutter: A program to cleave DNA with restriction enzymes. Nucleic Acids Res. 31:3688-3691.

Weisberg, S. 2005. Variable Selection. Pages 211-232 in: Applied Linear Regression, 3rd Ed., S. Weisberg, ed. John Wiley \& Sons, Hoboken, NJ.

WHEAT. 2014. Wheat: The vital grain of civilization and food security. In Wheat CRP Annual Report 2013. CGIAR, CIMMYT, Texcoco, Mexico. Available at: https://repository.cimmyt.org/xmlui/handle/10883/4016

Ye, K., Schulz, M. H., Long, Q., Apweiler, R., and Ning, Z. 2009. Pindel: A pattern growth approach to detect break points of large deletions and medium sized insertions from paired-end short reads. Bioinformatics 25: 2865-2871.
Zambino, P. J., Kubelik, A. R., and Szabo, L. J. 2000. Gene action and linkage of avirulence genes to DNA markers in the rust fungus Puccinia graminis. Phytopathology 90:819-826.

Zhang, N. W., Pelgrom, K., Niks, R. E., Visser, R. G. F., and Jeuken, M. J. W. 2009. Three combined quantitative trait loci from nonhost Lactuca saligna are sufficient to provide complete resistance of lettuce against Bremia lactucae. Mol. Plant-Microbe Interact 22:1160-1168.

\section{AUTHOR-RECOMMENDED INTERNET RESOURCES}

Babraham Bioinfomatics FastQC v0.11.2: https://www.bioinformatics.babraham.ac.uk/projects/fastqc

GitHub rust conversion scripts: https://github.com/umngao/rust_scores_conversion

Hannon Lab FASTX toolkit v.0.0.14:

http://hannonlab.cshl.edu/fastx_toolkit/index.html

New England Laboratories NEBcutter v2.0 software: http://nc2.neb.com/NEBcutter2/index.php

Phytozome 12 Brachypodium distachyon v3.1: https://phytozome.jgi.doe.gov

Primer3 tool: http://biotools.umassmed.edu/bioapps/primer3_www.cgi

SNP frequency graphing: https://github.com/ngs-docs/edda/blob/master/doc/tutorials-2012/files/plot allele_freq_data.R

United States Department of Energy Joint Genome Institute genome portal: http://genome.jgi.doe.gov/BradisBd23_FD/BradisBd23_FD.info.html

M. Vermaat's custom python script for syncing paired-end reads: https://github.com/martijnvermaat/bio-playground/tree/master/syncpaired-end-reads 